\title{
Sentinel biomarkers in HCV positive patients with mixed cryoglobulinemia
}

\author{
Umberto Basile $^{\mathrm{a}, *, 1}$, Mariapaola Marino ${ }^{\mathrm{b}, 1}$, Laura Gragnani ${ }^{\mathrm{c}}$, Cecilia Napodano ${ }^{\mathrm{d}, *}$, \\ Francesca Gulli ${ }^{\mathrm{e}}$, Krizia Pocino ${ }^{\mathrm{d}}$, Serena Lorini ${ }^{\mathrm{c}}$, Stefano Angelo Santini ${ }^{\mathrm{a}}$, Valerio Basile ${ }^{\mathrm{f}}$, \\ Luca Miele $^{\mathrm{d}}$, Anna Linda Zignego ${ }^{\mathrm{c}}$, Gian Ludovico Rapaccini ${ }^{\mathrm{d}}$ \\ a Fondazione Policlinico Universitario "A. Gemelli" - I.R.C.C.S, Università Cattolica del Sacro Cuore, Rome, Italy \\ b Istituto di Patologia generale, Fondazione Policlinico Universitario "A. Gemelli" - I.R.C.C.S, Università Cattolica del Sacro Cuore, Rome, Italy \\ ${ }^{\mathrm{c}}$ Interdepartmental Center for Systemic Manifestations of Hepatitis Viruses (MASVE), Department of Experimental and Clinical Medicine, and Department of Oncology, \\ Azienda Ospedaliero-Universitaria Careggi (AOUC), Florence, Italy \\ ${ }^{\mathrm{d}}$ Dipartimento di Medicina Interna e Gastroenterologia, Fondazione Policlinico Universitario "A. Gemelli" - I.R.C.C.S, Università Cattolica del Sacro Cuore, Rome, Italy \\ e Dipartimento di Medicina di Laboratorio, Ospedale Madre Giuseppina Vannini, Rome, Italy \\ ${ }^{\mathrm{f}}$ Dipartimento di Medicina di Laboratorio, Università di Tor Vergata, Rome, Italy
}

\section{A R T I C L E I N F O}

\section{Keywords:}

Hepatitis C virus

Mixed cryoglobulinemia

Free light chains

Vascular endothelial growth factor

Biomarkers

\begin{abstract}
A B S T R A C T
Background: Infections, autoimmunity and cancer play a role as determinants of etiology in Hepatitis C virus (HCV) related mixed cryoglobulinemia (MC). Several factors of risk have been suggested as markers of pathogenesis and progression of HCV-related MC into B cell Non-Hodgkin's Lymphoma (B-NHL). Here, we evaluated IgG subclass distribution, free light chains (FLCs) and vascular endothelial growth factor (VEGF) as a new combination of biomarkers.

Methods: We measured IgG1-4 subclasses, FLCs and VEGF levels in sera 53 from HCV-related MC, in comparison with 40 sera from HCV negative patients with rheumatoid arthritis (RA) and 30 from healthy blood donors (HBD).

Results: IgG3 levels were significantly higher in HCV-MC patients with a decrement of IgG2 and IgG4; FLC levels significantly increased in both MC and RA patients' groups; serological VEGF was higher in HCV-MC patients than in HBD in correlation with $\mathrm{k}$ and $\lambda$ levels.

Conclusion: Our results suggest that a specific IgG subclasses pattern together with raised levels of FLCs and VEGF could represent the biomarker "signature" of an inflammation multistage of acquired immune system.
\end{abstract}

\section{Introduction}

Chronic Hepatitis C virus (HCV) infection leads to the frequent association between liver and extrahepatic disorders. HCV tropism for lymphoid cells may contribute to poly-oligoclonal B-lymphocyte expansion causing the production of different autoantibodies and immunocomplexes, such as mixed cryoglobulins (CGs) that are commonly observed in a high proportion of patients with HCV infection (Zignego et al., 2017).

Mixed cryoglobulinemia (MC) consists in a systemic vasculitis characterized by the deposition of immunocomplexes composed of
HCV, anti-HCV polyclonal IgG, and monoclonal (type II) or polyclonal (type III) IgM with rheumatoid factor (RF) activity.

Infections, autoimmunity and cancer have been implicated as determinants of etiology in MC however; HCV is the most frequent infectious trigger (Gulli et al., 2017, Basile et al., 2018a, 2018b).

Due to the complex virus-host interactions, MC represents an excellent model to investigate the pathogenetic mechanisms of HCV infection and its evolution into autoimmune/lymphoproliferative disorders that are still poorly understood (Basile et al., 2018a, 2018b). The clinical spectrum of MC is extremely wide, ranging from completely asymptomatic forms without vasculitis to life-threatening

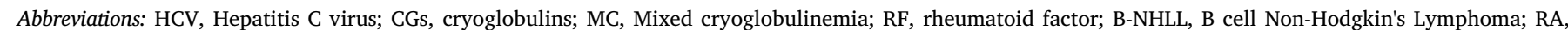

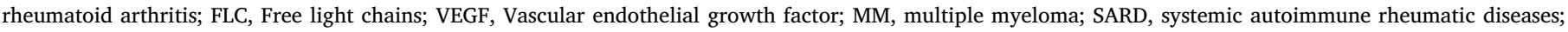
HBD, healthy blood donors; ELISA, enzyme-linked immunosorbent assay; DAA, direct-acting antiviral agents

*Corresponding author at: Fondazione Policlinico Universitario Agostino Gemelli IRCCS, Università Cattolica del Sacro Cuore, L.go A.Gemelli, 8, 00168 Rome, Italy.

E-mail addresses: umberto.basile@policlinicogemelli.it (U. Basile), cecilia.napodano@gmail.com (C. Napodano).

${ }^{1}$ Umberto Basile and Mariapaola Marino equally contributed to this work. 
complications; the worsening evolution into B cell Non-Hodgkin's Lymphoma (B-NHL) occurs in about $10 \%$ of cases (Ramos-Casals et al., 2012).

Human IgG class includes four subclasses (IgG1-4), highly conserved, that differ in their constant region, particularly in their hinges and upper $\mathrm{CH} 2$ domains. Although they are $>90 \%$ identical on the amino acid level, each subclass has a unique profile with respect to antigen binding, immunocomplexes formation, complement activation, triggering of effector cells, half-life, and placental transport. Differences in structure at the hinge region, where the Fab section bends and rotates with respect to the Fc section determines the differential ability to bind their target; the flexibility at the hinge is greatest in IgG3 (Sigal, 2012). Only recently, a different IgG subclasses distribution in serum between patients with autoimmune diseases and healthy controls has been described, with distinct patterns in different conditions (Zhang et al., 2015). A higher prevalence of IgG3 subclass in responses to HCV antigens seems to be more frequent in those patients who are HCV- and MC-positive, rather than in patients who are HCV-positive and MCnegative (Basile et al., 2017a, 2017b, Dispenzieri and Gorevic, 1999). Human and murine IgG3 display an equivalent tendency to self-assemble, although there are structural and functional differences, demonstrating a role in the molecular mechanism of the formation of cryoprecipitate (Otani et al., 2012; Izui et al., 1993). In the years, the study and characterization of human CGs in patients with HCV infection showed a prevalent abundance of IgG3 in cryoprecipitates suggesting that the immune system is highly stimulated and more prone to the development of autoimmune disorders, supporting the hypothesis of a specific involvement of IgG3 subclass as an early trigger of CGs (Basile et al., 2017a, 2017b). Recently, we reported the evidence of CGs exclusively composed of IgG1 and IgG3 with RF activity, maybe representing an early stage of MC (Gulli et al., 2018). Immunoglobulin(Ig-) free light chains (FLCs) are bioactive molecules (Basile et al., 2017b) produced during the synthesis of immunoglobulins. Due to their fast turnover, particularly for the $\mathrm{k}$ chain $(<6 \mathrm{~h}$ compared to 20-25 days to total IgG), and in subjects with normal kidney function, serum levels could be considered as a direct marker of B-cell activity, which is otherwise difficult to measure in routine clinical practice. Different reports and guidelines demonstrated the relevance of serological FLC levels in the diagnostic and prognosis pathway of diseases caused by deposition of immunocomplexes and immune-proliferative disorders, and for monitoring the therapeutic outcomes (Basile et al., 2017b; Charafeddine et al., 2012; Graziani and Merlini, 2014). A serological increase of polyclonal FLC may help to identify individuals at higher risk of developing a monoclonal gammopathy. Moreover, polyclonal increments may anticipate the development of MGUS, suggesting their role as determinant of pathogenesis (Kumar et al., 2019). The assessment of FLCs and the $\mathrm{k} / \lambda$ ratio in patients with HCV infection may be clinically useful especially for predicting the onset of MC. Terrier and co-workers described high levels of free $\mathrm{k}$ chains in HCVpositive patients with MC and type II MC (Terrier et al., 2009). An abnormal $\mathrm{k} / \lambda$ ratio may be useful to predict response to biological drugs that represent an expensive treatment for patients with HCV-related MC (Basile et al., 2015).

Vascular endothelial growth factor (VEGF) represents a primary driving force for both physiological and pathological angiogenesis, and raised circulating VEGF levels have been described in lymphoproliferative disorders as multiple myeloma (MM), chronic myeloid leukemia, chronic myelomonocytic leukemia, chronic lymphocytic leukemia, and lymphoma, often in correlation with disease activity (Pihan et al., 2018; Villani et al., 2016). VEGF plays a vital role in the progression of NHL and a systemic meta-analysis study showed that the overexpression of VEGF in lymphoma tissue represents a promising potential prognostic factor in NHL (Yang et al., 2015). The fibrogenic effect of VEGF through multiple mechanisms is well known, including the promotion of inflammation, and the release of fibrosis enhancing molecules (Lee et al., 2004; Yoshiji et al., 2003). Of note, serum VEGF levels are significantly increased also in patients with systemic autoimmune rheumatic diseases (SARD) (Choi et al., 2003) with serological levels correlated to disease severity and clinical complications (Hashimoto et al., 2003).

In this study, we measured the serological levels of IgG subclasses, FLCs, and VEGF in 53 HCV-related MC patients compared to $40 \mathrm{HCV}$ negative patients with active RA and to healthy blood donors (HBD). Our aim was to verify if this new combination of biomarkers may be useful to evaluate the progression of inflammation stage.

\section{Materials and methods}

\subsection{Patients and controls}

Ninety-three patients with chronic diseases were retrospectively enrolled including $53 \mathrm{HCV}$ related MC (median age 60.4 \pm 1.7 years) and $40 \mathrm{HCV}$-negative patients with RA (median age $62 \pm 5.0$ years); 30 HBD (median age $60 \pm 7.2$ years) were used as negative controls.

Patients were enrolled at two Italian centers (Center for Systemic Manifestations of Hepatitis Viruses (MaSVE), Department of Experimental and Clinical Medicine, University of Florence, Florence; Fondazione Policlinico Agostino Gemelli- I.R.C.C.S, Università Cattolica del Sacro Cuore, Rome). The main demographic, clinical, and virological characteristics of the study population are reported in Table 1.

HCV-related MC patients were included in the study according to the following criteria: presence of HCV-RNA in serum; positivity for type II CG; absence of antiviral treatment and/or immunosuppressive therapy; presence of MC symptoms, according to the classification criteria for MC as proposed by the Italian Group for the Study of Cryoglobulinemias in 1989 and later revised in 2002 (Ferri et al., 2002).

Transient elastography represents a very useful and advantageous diagnostic tool because it can be performed repeatedly, does not require a highly experienced operator, and has a low risk of complications. Measurements of liver stiffness with transient elastography in these patients are well correlated with fibrosis METAVIR stages.

The inclusion criteria for the $40 \mathrm{HCV}$-negative RA patients were represented by absence of radiologically visible lesions, absence of therapy, presence of a high level of anti-cyclic citrullinated peptide, positivity for rheumatoid factor.

All patients had a normal renal function with an estimated

Table 1

Demographic, clinical, and virological features correlates of HCV-related MC patient.

\begin{tabular}{ll}
\hline & MC Patients $(n=53)$ \\
\hline Age(Years) & $60.4( \pm 1.7)$ \\
Gender(Male/Female) & $29 / 24$ \\
Metavir score & \\
F0-F1 & 0 \\
F2 & 0 \\
F3 & 23 \\
F4 & 30 \\
Child Pugh-score (A/B/C) & $28 / 2 / 0$ \\
ALT (U/L) & $120.4( \pm 10)$ \\
AST (U/L) & $94( \pm 8)$ \\
Total Bilirubin (mg/dL) & $0.94( \pm 0.05)$ \\
Direct Bilirubin (mg/dL) & $0.32( \pm 0.02)$ \\
HCV-RNA (IU/mL $\left.\times 10^{6}\right)$ & $2.7( \pm 0.48)$ \\
HCV genotype: & \\
1a & $8(14.8 \%)$ \\
1b & $32(61.1 \%)$ \\
2 & $4(7.4 \%)$ \\
3 & $7(13 \%)$ \\
4 & $2(3.7 \%)$ \\
Cryocrit (\%) & $4.2( \pm 0.2)$ \\
RF (UI/mL) & $30.5( \pm 1.7)$ \\
C4 (mg/dL) & $0.15( \pm 0.02)$ \\
& \\
\hline
\end{tabular}


glomerular filtration rate of $\geq 60 \mathrm{~mL} / \mathrm{min} / 1.73 \mathrm{~m} 2$.

The whole study was conducted according to the Declaration of Helsinki and approved by the Ethical Committee at the Università Cattolica; all the participants provided written informed consent prior to enrollment. All samples were processed anonymously.

\subsection{Laboratory testing}

Sera were obtained by standard centrifugation, divided into aliquots and stored frozen until analysis. Samples were thawed only once and immediately assayed in a blinded fashion and in a single batch. FLCs were assessed using the Freelite ${ }^{\mathrm{TM}}$ Human Kappa and Lambda Free Kits (The Binding Site, Birmingham, UK) on an Optilite instrument (The Binding Site, Birmingham, UK; free $\kappa$ normal range: $3.3-19.4 \mathrm{mg} / \mathrm{L}$; free $\lambda$ normal range: $5.7-26.3 \mathrm{mg} / \mathrm{L}$ ). A ratio of $\mathrm{k} / \lambda<0.26$ or $>1.65$ is abnormal, according to the manufacturer's recommendations.

The four IgG subclasses concentrations were measured by turbidimetry through the employment of Human IgG and IgG subclass liquid reagent kits (The Binding Site Birmingham, UK) with Optilite instrument according to the manufacturer's recommendations. These kits are intended for quantifying human IgG and IgG subclasses. Concentrations are automatically calculated by reference to a standard curve stored within the instrument. Normal range for subclasses: $3.82-9.29 \mathrm{~g} / \mathrm{L}$ for IgG1; $2.42-7.0 \mathrm{~g} / \mathrm{L}$ for IgG2; $0.22-1.76 \mathrm{~g} / \mathrm{L}$ for IgG3; $0.04-0.86 \mathrm{~g} / \mathrm{L}$ for IgG4.

Samples were tested according to the manufacturer's instructions and serum dilutions, where necessary, were performed according to the manufacturer's recommendations.

Assessment of serum VEGF levels was performed through the enzyme-linked immunosorbent assay (ELISA) employing monoclonal antihuman VEGF antibodies (R\&D System, Minneapolis, MN, USA). The detectable concentration ranges were $31.2-2000 \mathrm{pg} / \mathrm{mL}$ for VEGF.

Anti-cyclic Citrullinated Peptide and rheumatoid factor were detected through a magnetic microparticle based method using chemiluminescent detection (acridinium) to provide a high degree of precision and rapidity of analysis (Menarini Diagnostics Zenit, Bologna, Italy). Samples were tested according to the manufacturer's instructions and serum dilutions, where necessary, were performed according to the manufacturer's recommendations.

HCV-RNA was assessed in both supernatant and cryoprecipitate and was determined through real-time polymerase chain reaction, transcription-mediated amplification, and multi-probe reverse hybridization of the $5^{\prime}$ untranslated region of the HCV genome. The HCV genotype was determined for each sample by using the line probe assay Versant HCV genotype LiPA 2.0 (Siemens Healthcare Diagnostics, Munich, Germany). Genotypes and subtypes detectable from this test include $1,1 \mathrm{a}, 1 \mathrm{~b}, 2,2 \mathrm{a} / 2 \mathrm{c}, 2 \mathrm{~b}, 3,3 \mathrm{a}, 3 \mathrm{~b}, 3 \mathrm{c}, 4,4 \mathrm{a}, 4 \mathrm{~b}, 4 \mathrm{c} / 4 \mathrm{~d}, 4 \mathrm{e}, 4 \mathrm{f}, 4 \mathrm{~h}$, $5 \mathrm{a}$, and $6 \mathrm{a}$.

All the determinations were performed by an operator without knowledge of the clinical information of the handled sample. Each sample was tested twice to minimize eventual discrepancies, and all tests were performed in the same laboratory with the same instruments. All safety precautions were taken while handling potentially harmful samples.

\subsection{Statistical analysis}

Comparison of mean values was performed by Student's $t$-test; $p$ values $<.05$ were considered significant. A correlation analysis was carried out using the Pearson correlation coefficient.
Table 2

IgG subclasses distribution in patients and controls.

\begin{tabular}{llll}
\hline & HCV & RA & HBD \\
\hline $\mathrm{IgG1}(\mathrm{g} / \mathrm{L})$ & $7.34 \pm 2.91$ & $8.71 \pm 4.48$ & $7.00 \pm 1.62$ \\
$\mathrm{IgG} 2(\mathrm{~g} / \mathrm{L})$ & $2.77 \pm 1.44^{*}$ & $4.07 \pm 2.20$ & $4.00 \pm 1.38$ \\
$\mathrm{IgG3}(\mathrm{g} / \mathrm{L})$ & $1.01 \pm 0.42^{* *}$ & $0.88 \pm 0.54$ & $0.68 \pm 0.33$ \\
$\mathrm{IgG4}(\mathrm{g} / \mathrm{L})$ & $0.31 \pm 0.20^{* *}$ & $0.55 \pm 0.47$ & $0.61 \pm 0.58$ \\
\hline
\end{tabular}

MC: mixed cryoglobulinemia. RA: rheumatoid arthritis. HBD: healthy blood donors.

Data are expressed as mean \pm standard error.

Normal range for subclasses: $3.82-9.29 \mathrm{~g} / \mathrm{L}$ for IgG1; 2.42-7.0 g/L for IgG2; $0.22-1.76 \mathrm{~g} / \mathrm{L}$ for $\mathrm{IgG} 3 ; 0.04-0.86 \mathrm{~g} / \mathrm{L}$ for $\mathrm{IgG} 4$.

$p<.05$.

$p<.01$

\section{Results}

3.1. Serological IgG subclasses distribution among HCV-related $M C, R A$ patients and $H B D$

The mean values ( \pm standard deviation) of IgG subclasses are reported in Table 2 and displayed in Fig. 1A. Only in HCV-related MC patients, serum IgG subclasses showed a significantly different distribution from HBD even if the mean values were within the normal range. In particular, IgG3 were higher in HCV patients than in HBD $(1.01 \pm 0.42 \mathrm{~g} / \mathrm{L}$ vs $0.68 \pm 0.33 \mathrm{~g} / \mathrm{L}, p<.01)$; on the contrary, IgG2 and IgG4 were lower in HCV-positive patients if compared to HBD (IgG2: $2.77 \pm 1.44 \mathrm{~g} / \mathrm{L}$ in HCV vs $4.00 \pm 1.38 \mathrm{~g} / \mathrm{L}$ in HBD, $p<.05$; IgG4: $0.31 \pm 0.20 \mathrm{~g} / \mathrm{L}$ in HCV vs $0.61 \pm 0.58 \mathrm{~g} / \mathrm{L}$ in $\mathrm{HBD}, p<.01$ ).

\subsection{Serological FLCs levels in HCV-related MC, RA-patients and HBD}

The mean values ( \pm standard deviation) of FCLs and $\mathrm{k} / \lambda$ ratio are reported in Table 3 and visualized in Fig. 1B. The statistical analysis revealed significant differences between patients and HBD. The mean values of $\mathrm{k}$ and $\lambda$ free chain levels were significantly higher in both patients' groups (HCV-MC $\mathrm{k}$ and $\lambda$ : $25.94 \pm 11.54 \mathrm{mg} / \mathrm{L}$ and $34.63 \pm 20.44 \mathrm{mg} / \mathrm{L}$, respectively; RA $\mathrm{k}$ and $\lambda: 35.89 \pm 35.87 \mathrm{mg} / \mathrm{L}$ and $22.58 \pm 23.90 \mathrm{mg} / \mathrm{L}$, respectively) if compared to HBD (HBD k: $8.88 \pm 3.86 \mathrm{mg} / \mathrm{L}$, HBD $\lambda: 12.90 \pm 7.05 \mathrm{mg} / \mathrm{L}$ ). FLCs mean values were above the positivity cut-off for HCV-MC patients while in RA free $\lambda$ mean value was still within the normal range (as determined by the manufacturer); for this reason, the $\mathrm{k} / \lambda$ ratio was significantly higher only in RA patients when compared to HBD $(1.73 \pm 0.59$ vs $0.75 \pm 0.21$, respectively).

\subsection{Serum VEGF assessment in HCV-related MC patients and HBD}

The mean value of VEGF in HCV-related MC patients was higher and statistically more significant than in HBD $(441.78 \pm 185.78 \mathrm{pg} / \mathrm{mL}$ vs $64.85 \pm 29.45 \mathrm{pg} / \mathrm{mL}, p<.01$ ), as shown in Fig. 1C. We evaluated if VEGF correlated with FLCs levels in MC patients. We found that VEGF displayed a correlation with both free chains that was strong with free $\mathrm{k}$ $(R=0.706383828)$ and moderate with free $\lambda(R=0.52102002)$ (Fig. 1D).

\section{Discussion}

HCV driven autoimmune and lymphoproliferative disorders are the consequence of a multifactorial and multistep pathogenetic process and HCV-related MC represents the prototype of a complex intersection between autoimmunity and neoplastic disorders (Saadoun et al., 2007). Because CGs producing B-cells in the course of HCV infection are mostly monoclonal, HCV-related MC can be considered a benign B-cell lymphoproliferative condition. The overall risk of non-Hodgkin's 

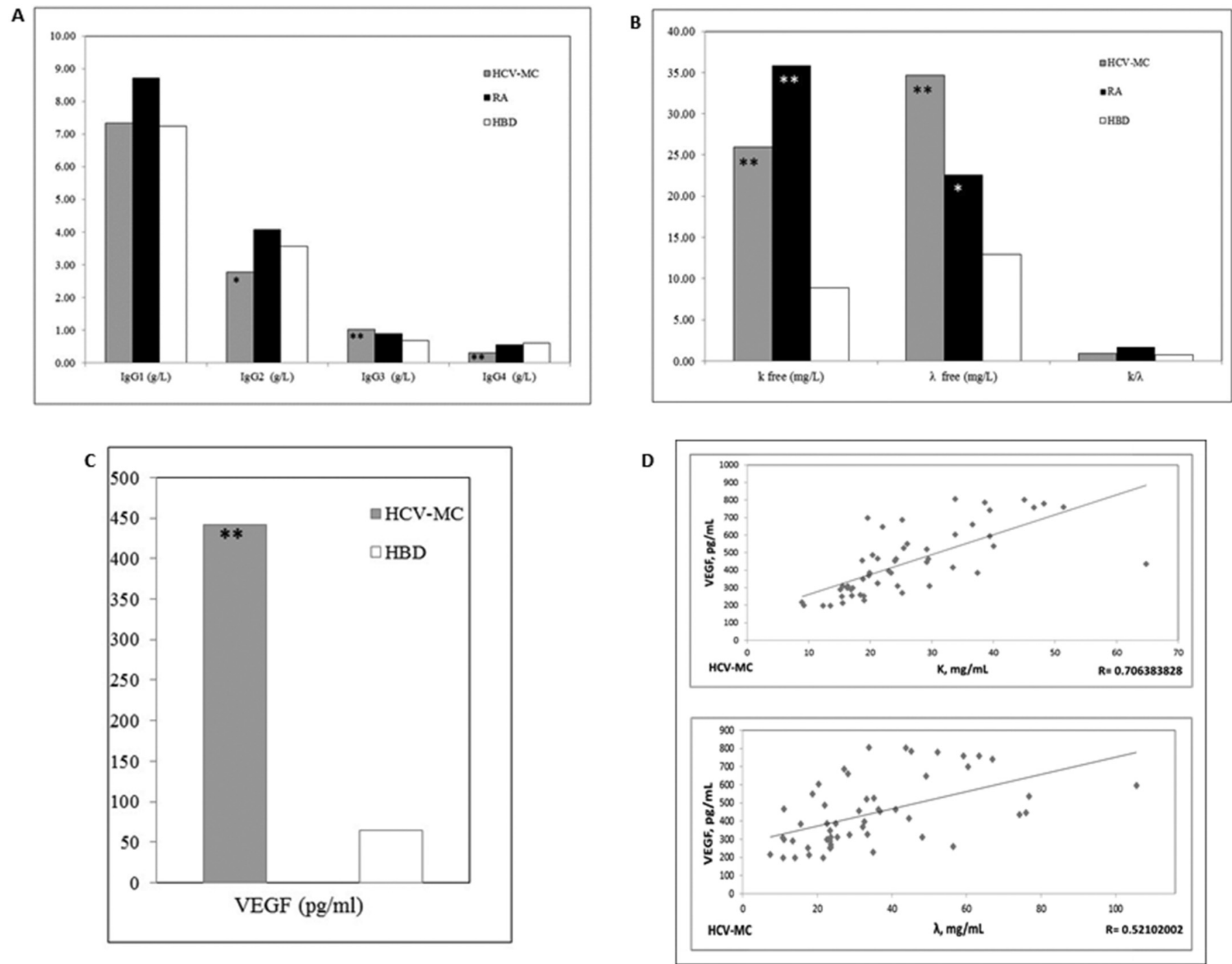

Fig. 1. Distribution of IgG Subclasses, FLC and VEGF in patients and control group.

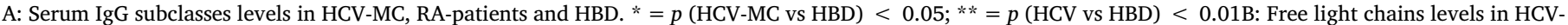

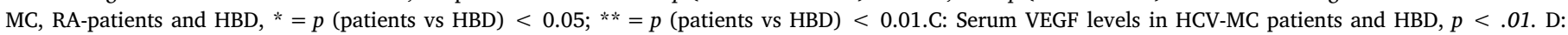
Correlation between $\mathrm{k}$ and $\lambda$ levels and VEGF in HCV-MC patients.

lymphoma in patients with HCV-MC is estimated to be 35 times higher than in the general population (Gulli et al., 2017). The mechanisms underlying the development of HCV-related B-cell lymphoma remain to be elucidated. Until now, limited data concerning a direct infection of B-cells in HCV-associated B-cell lymphoma are reported in literature (Levine et al., 2003). Alternatively, HCV can exert its oncogenic potential indirectly through chronic stimulation on the immune system, leading to the selection of abnormal B clones.

Viral infections usually lead to the increase of $\operatorname{IgG1}$ and IgG3 subclasses, with the IgG3 switching first appearing during the anti-viral immune response. In a previous paper, by the employment of immunofixation, we reported that IgG3 monoclonal CGs with RF activity induced extensive extra-hepatic manifestations in HCV patients (Basile et al., 2017a, 2017b, Basile et al., 2018b). In the present study, we confirmed our results on IgG3 subclass as initial trigger of CGs formation by using a turbidimetric assay that allows the quantification of human IgG subclasses. The measurement of IgG subclasses is a valuable tool in the diagnosis of abnormal protein metabolism and immune

Table 3

$\mathrm{k}, \lambda$ and $\mathrm{k} / \lambda$ ratio mean values in patients and controls.

\begin{tabular}{lll}
\hline & $\kappa f$ free $(\mathrm{mg} / \mathrm{L})$ & $\lambda$ free $(\mathrm{mg} / \mathrm{L})$ \\
\hline HCV & $25.94 \pm 11.54(\mathrm{p}<.01)$ & $34.63 \pm 20.44(\mathrm{p}<.01)$ \\
RA & $35.89 \pm 35.87(\mathrm{p}<.01)$ & $22.58 \pm 23.90(\mathrm{p}<.05)$ \\
HBD & $8.88 \pm 3.86$ & $12.90 \pm 7.05$
\end{tabular}

MC: mixed cryoglobulinemia. RA: rheumatoid arthritis. HBD: healthy blood donors. NS: Not Significant.

Data are expressed as mean \pm standard error;

Normal range for FLCs: $3.30-19.40 \mathrm{mg} / \mathrm{L}$ for $\mathrm{k}$; $5.71-26.30 \mathrm{mg} / \mathrm{L}$ for $\lambda$. A ratio of $\mathrm{k} / \lambda<0.26$ or $>1.65$ is abnormal;

p was calculated between each patient group and HBD. 
system's lack ability to face infectious agents. Moreover, we explored the serological concentration distribution of Ig subclasses in $53 \mathrm{HCV}$ related MC patients in comparison to $40 \mathrm{HCV}$ negative patients with a systemic autoimmune disorder such as RA and with $30 \mathrm{HBD}$. We found that levels of serum IgG3 subclass were higher in HCV-MC patients than in RA and HBD (in the latter case, with a statistically significant difference), confirming our previous observation about high presence of IgG3 in cryoprecipitate (Basile et al., 2017a, 2017b). The different profile of IgG subclasses distribution that we found in HCV-MC could reflect a different immune response depending on the specific autoantigen and time of stimulation. The evidence of an association with a specific IgG subset increase was crucial to the identification of IgG4related diseases. However, the possibility of other associations between autoimmune diseases and IgG subclasses are still less investigated (Kamisawa et al., 2015). It is also conceivable that, for the same IgG subclass, different cut-offs may be considered for different diseases.

Our results strengthen the hypothesis that IgG3 subclass increase represents the first alert step towards the pathogenesis of CGs and autoimmune response in these patients. Subsequently early intervention of FLC at the initial phase might modify natural history of the disease in a negative way. This is likely to lead to screening approach and identification of early state of CGs in the population with HCV infection (Fig. 2A) (Basile et al., 2017a, 2017b).

The synthesis of antibodies is accompanied by an excess of light chain production giving rise to a release of about $500 \mathrm{mg} /$ day of polyclonal FLC in the peripheral circulation (Basile et al., 2017b). This production is abnormally higher in several pathological autoimmune conditions reflecting B-cell activation and giving insight into the activity of the adaptive immune system. Particularly, FLCs may represent a marker of overall B-cell activity in those diseases where there is an increase of autoantibodies and immune complexes such as SARD (Napodano et al., 2019). In these conditions, characterized by chronic inflammatory reactions, FLCs may play a pathogenetic role. Owing to their short half-life, FLC quantitative analysis has become a useful diagnostic tool to measure B clonal expansion in plasma cell dyscrasias. Specific FLC patterns were also associated with the presence of MC vasculitis and/or B-NHL in HCV-positive patients with an interesting correlation to antiviral therapy response (Geri et al., 2010). Clinical interest in the determination of FLCs in viral infections (human immunodeficiency virus or hepatitis $\mathrm{C}$ virus) derives from their putative role as an early biomarker of tumor onset, especially lymphoma (Bibas et al., 2012). MC and B-NHL represent the main extrahepatic manifestations of HCV (Gragnani et al., 2015) and serum levels of FLCs have recently been used as marker of worsening progression (Oliveira et al., 2014). In a previous report, analyzing a group of HCV-positive patients with/without MC, we clearly confirmed the role of FLC as a serum biomarker of disease activity, and of rituximab therapy response (Basile et al., 2015). In the present study, we analyzed the FLC serological profile in a larger group of HCV-MC patients in comparison to HCVnegative patients with RA, a systemic autoimmune disorder characterized by a strong elevation of circulating FLCs levels. Our results confirm that HCV-related MC is strongly associated with high levels of FLCs comparable to RA, underlying the strong correlation with B-cell hyperactivity in both diseases. Specific FLC patterns were also associated with MC vasculitis and/or B-NHL in HCV-positive patients with an interesting correlation to antiviral therapy response (Gragnani et al., 2015; Geri et al., 2010). We hypothesize that following HCV exposure, B-lymphocytes could be activated to produce complete Ig, and polyclonal FLCs. FLCs could bind B cell surface through a still unknown receptor and become "key" mediators of the inflammatory response (Fig. 2B).

Extrahepatic manifestations in HCV-positive patients involve multiple organ systems with a variety of comorbidities, which may lead to significantly increased mortality rate. VEGF is the most important
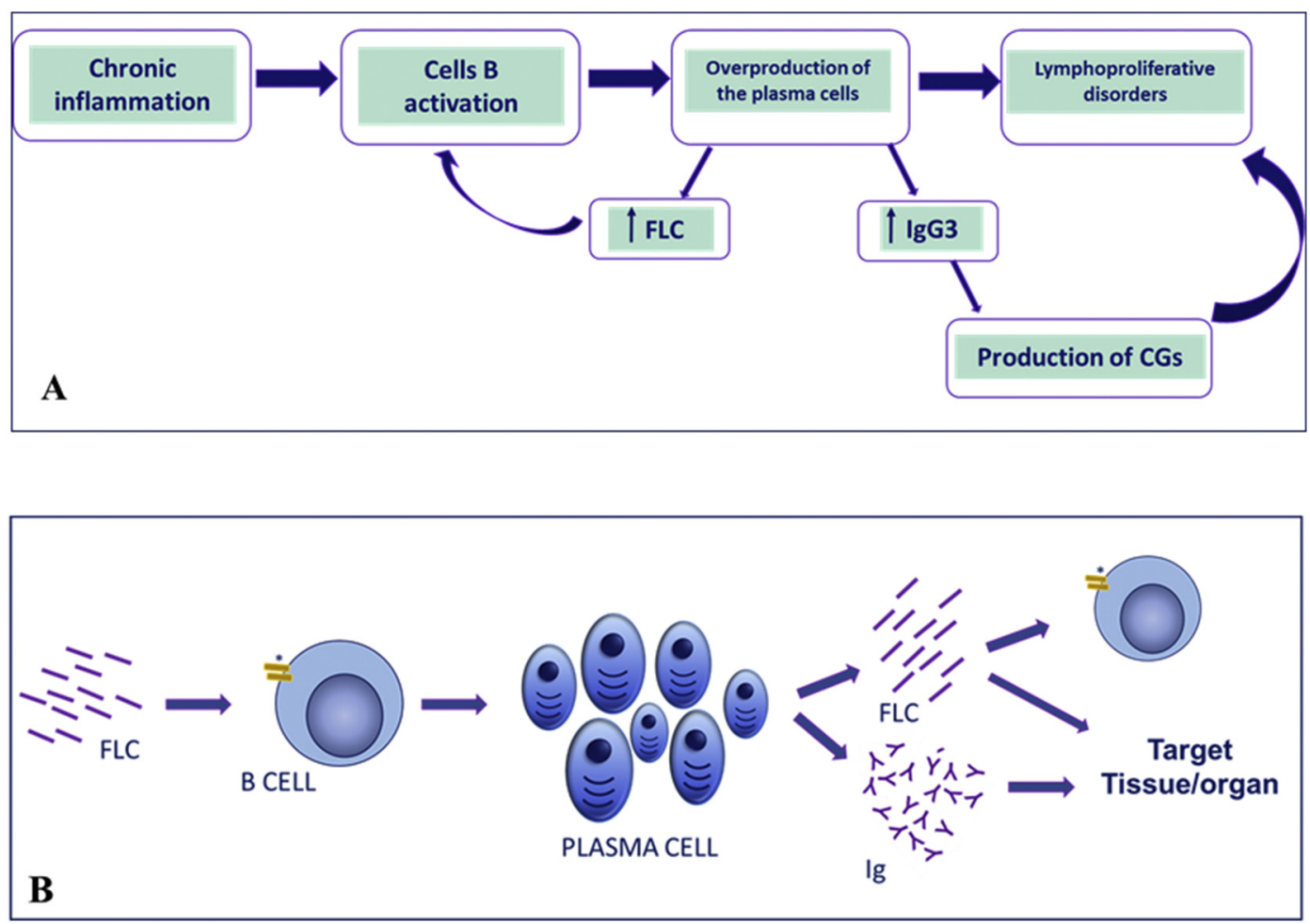

Fig. 2. Activation of innate immunity. A: the prolonged stimulation of the immune system, during the inflammatory process, can lead to hyperstimulation of plasma cells and polyclonal activation of B cells. Activated B cells differentiate into plasma cells and produce IgG3 autoantibodies directed against tissue and immunoglobulin autoepitopes. IgG3 subclass is involved in the initiation of the early stages of CGs. The overproduction of plasma cells causes a considerable increase of the polyclonal Free Light Chains (FLC) concentration, which could be responsible for further cell activation. B: FLCs through the binding of a B cell surface, still unknown, receptor $(*)$ could mediate the inflammatory respon. 
determining factor for both physiological and pathological angiogenesis. It plays an important role in the induction of neovascularization in solid cancers contributing to the invasive ability of liver tumors, especially of hepatocellular carcinoma (Lee et al., 2004, Clauss, 2000, Mukozu et al., 2013); raised levels of VEGF have been described also in different hematologic malignancies. Hashimoto and co-workers showed its increase in SARD patients with serum levels correlated with disease severity and the presence of complications. Here, we analyzed VEGF serological levels in HCV-related MC patients: we found that not only it was higher and statistically more significant than HBD $(441.78 \pm 185.78 \mathrm{pg} / \mathrm{mL}$ vs $64.85 \pm 29.45 \mathrm{pg} / \mathrm{mL})$, but also higher if compared to the mean value described for RA patients $(359 \pm 94 \mathrm{pg} /$ $\mathrm{mL}$ by Hashimoto and coworkers) (Hashimoto et al., 2003). Moreover, the finding of a positive correlation of raised VEGF levels with both $\mathrm{k}$ and $\lambda$ FLCs in HCV-MC patients could be suggestive that the strong viral-driven $B$ activation would be worsening into lymphoproliferative disorders (Fig. 1D).

In conclusion, our main goal is to identify a biomarker profile that could be very helpful in patients' management and as screening test, for setting diagnosis and classification of patients with HCV-related MC. Precision medicine approach is the start of routine care for most patients. Based on our results, we suggest that the worsening progression of disease towards extrahepatic manifestations could be monitored with specific IgG profile together with increased serological levels of FLCs and VEGF. Following further validation, their employment in new integrated specific diagnostic panels will represent the main challenge of clinical and laboratory investigations. Further studies will be useful to better clarify the role of VEGF and FLC in the inflammatory multi-stadial progression in lymphoma.

\section{Funding}

This research and its publication have been funded by Università Cattolica del Sacro Cuore as part of its programs on the promotion and dissemination of scientific research (Linea D1 to Mariapaola Marino) and by the "Associazione Italiana per la Ricerca sul Cancro" (AIRC) (Investigator Grant Id.17391), "Istituto Toscano Tumori" (ITT), "Ente Cassa di Risparmio di Firenze" and "Fondazione Oretta Bartolomei Corsi".

\section{Disclosure statement}

The other authors declare that they do not have anything to disclose with regard to funding or conflict of interest concerning this manuscript.

\section{Acknowledgements}

The authors would like to thank Ms. Helena Ritchie for her kind language editing. Laura Gragnani and Anna Linda Zignego gratefully acknowledges BMS and Gilead Sciences (Merck, AbbVie, Janssen-Cilag) for consulting and lecturing fees.

\section{References}

Basile, U., et al., 2015. Assessment of free light chains in HCV-positive patients with mixed cryoglobulinaemia vasculitis undergoing rituximab treatment. Liver Int. 35, $2100-2107$.

Basile, U., et al., 2017a. IgG3 subclass: a possible trigger of mixed cryoglobulin cascade in hepatitis C virus chronic infection. Dig. Liver Dis. 49, 1233-1239.
Basile, U., et al., 2017b. Free light chains: eclectic multipurpose biomarker. J. Immunol. Methods 451, 11-19.

Basile, U., et al., 2018a. Serological profile of asymptomatic HCV positive patients with low level of cryoglobulins. Biofactors 45, 318-325.

Basile, U., et al., 2018b. Different biochemical patterns in type II and type III mixed cryoglobulinemia in HCV positive patients. Dig. Liver Dis. 50 (9), 938-943.

Bibas, M., et al., 2012. Role of serum free light chains in predicting HIV-associated nonHodgkin lymphoma and Hodgkin's lymphoma and its correlation with antiretroviral therapy. Am. J. Hematol. 87, 749-753.

Charafeddine, K.M., et al., 2012. Extended use of serum free light chain as a biomarker in lymphoproliferative disorders: a comprehensive review. Am. J. Clin. Pathol. 137, 890-897.

Choi, J.J., et al., 2003. Elevated vascular endothelial growth factor in patients with systemic sclerosis. J. Rheumatol. 30, 1529-1533.

Clauss, M., 2000. Molecular biology of the VEGF and the VEGF receptor family. Semin. Thromb. Hemost. 26, 561-569.

Dispenzieri, A., Gorevic, P.D., 1999. Cryoglobulinemia. Hematol.Oncol. Clin. N. Am. 13, 1315-1349.

Ferri, C., Zignego, A.L., Pileri, S.A., 2002. Cryoglobulins. J. Clin. Pathol. 55, 4-13.

Geri, G., et al., 2010. Surrogate markers of B cell non-Hodgkin's lymphoma in patients with hepatitis C virus-related cryoglobulinaemia vasculitis. Ann. Rheum. Dis. 69, 2177-2180.

Gragnani, L., et al., 2015. Long-term effect of HCV eradication in patients with mixed cryoglobulinemia: a prospective, controlled, open-label, cohort study. Hepatology 61, 1145-1153.

Graziani, M.S., Merlini, G., 2014. Serum free light chain analysis in the diagnosis and management of multiple myeloma and related conditions. Expert. Rev. Mol. Diagn. $14,55-66$.

Gulli, F., et al., 2017. Cryoglobulin test and cryoglobulinemia hepatitis C-virus related. Mediterr. J. Hematol. Infect. Dis. 9, e2017007.

Gulli, F., et al., 2018. IgG cryoglobulinemia. Eur. Rev. Med. Pharmacol. Sci. 22, 6057-6062.

Hashimoto, N., et al., 2003. Levels of vascular endothelial growth factor and hepatocyte growth factor in sera of patients with rheumatic diseases. Mod. Rheumatol. 13 (2), 129-134.

Izui, S., et al., 1993. IgG3 CGs in autoimmune MRL-lpr/lpr mice: immunopathogenesis, therapeutic approaches and its relevance to similar human diseases. Ann. Rheum. Dis. 52 (Suppl. 1), S48-S54.

Kamisawa, T., et al., 2015. IgG 4-related disease. Lancet 385, 1460-1471.

Kumar, S., et al., 2019. Polyclonal serum free light chain elevation is associated with increased risk of monoclonal gammopathies. Blood Cancer J. 9, 49.

Lee, C.G., et al., 2004. Vascular endothelial growth factor (VEGF) induces remodeling and enhances TH2-mediated sensitization and inflammation in the lung. Nat. Med. 10, 1095-10103.

Levine, A.M., Shimodaira, S., Lai, M.M., 2003. Treatment of HCV-related mantle-cell lymphoma with ribavirin and pegylated interferon alfa. N. Engl. J. Med. 349, 2078-2079.

Mukozu, T., et al., 2013. Serum VEGF as a tumor marker in patients with HCV-related liver cirrhosis and hepatocellular carcinoma. Anticancer Res. 33, 1013-1021.

Napodano, C., et al., 2019. Free light chains and autoimmunity. Autoimmun. Rev. 18, 484-492.

Oliveira, I.S., et al., 2014. Serum levels of immunoglobulin free light chains in patients with chronic hepatitis C presenting cryoglobulinemia. Braz. J. Infect. Dis. 18, 638-642.

Otani, M., et al., 2012. Sialylation determines the nephrito genicity of IgG3 CGs. J. Am. Soc. Nephrol. 23, 1869-1878.

Pihan, M., et al., 2018. Raised VEGF: high sensitivity and specificity in the diagnosis of POEMS syndrome. Neurology 15 (5), e486.

Ramos-Casals, M., et al., 2012. The cryoglobulinaemias. Lancet 379, 348-360.

Saadoun, D., et al., 2007. Hepatitis C-associated mixed cryoglobulinaemia: a crossroad between autoimmunity and lymphoproliferation. Rheumatology (Oxford) 46, 1234-1242.

Sigal, L.H., 2012. Basic science for the clinician 58: IgG subclasses. J. Clin. Rheumatol. 18, 316-318.

Terrier, B., et al., 2009. Serum free light chain assessment in hepatitis C virus-related lymphoproliferative disorders. Ann. Rheum. Dis. 68, 89-93.

Villani, R., et al., 2016. DAAs rapidly reduce inflammation but increase serum VEGF level: a rationale for tumor risk during anti-HCV treatment. PLoS One 11, e0167934.

Yang, J., et al., 2015. VEGF overexpression is a valuable prognostic factor for nonHodgkin's lymphoma evidence from a systemic meta-analysis. Dis. Markers 2015, 786790.

Yoshiji, H., et al., 2003. Vascular endothelial growth factor and receptor interaction is a prerequisite for murine hepatic fibrogenesis. Gut 52, 1347-1354.

Zhang, H., et al., 2015. Serum IgG subclasses in autoimmune diseases. Medicine 94, e387.

Zignego, A.L., et al., 2017. International therapeutic guidelines for patients with HCV related extrahepatic disorders. A multidisciplinary expert statement. Autoimmun. Rev. 16, 523-541. 